\title{
Coding GPCR-G protein specificity
}

\author{
Cell Research (2017) 27:1193-1194. doi:10.1038/cr.2017.92; published online 11 July 2017
}

G protein-coupled receptors (GPCRs) are ubiquitous gatekeepers of cellular response and signal predominantly by recruitment and activation of $\mathbf{G}$ proteins. In a recent paper in Nature, Flock et al. use large-scale bioinformatics to build a model of GPCR-G protein selectivity and an interactive database to interrogate potential receptor- $G$ protein interactions.

G protein-coupled receptors (GPCRs) are the largest family of cell surface proteins with more than 800 members in the human genome, recognizing over 1000 different ligands. In contrast, the primary GPCR signaling partners, the $\alpha$ subunits of heterotrimeric $\mathrm{G}$ proteins, are encoded by only 16 human genes. Specific GPCR-G protein coupling is essential for normal physiology but the broad overlap in GPCR and G-protein expression imply that protein-protein recognition specificities must exist to enable coupling specificity. Previous efforts to decode GPCR-G protein selectivity have not been generally applicable across the GPCR superfamily. Flock et al. [1] take a large-scale bioinformatics approach, integrating sequence data across distantly related Opisthokonta along with structural and known mutagenesis data to develop both a model of GPCR-G protein selectivity and a useful interactive database that will help guide research in this field.

GPCRs can be considered as membrane-spanning guaninenucleotide exchange factors (GEFs) for heterotrimeric $G$ proteins. They are regulated by ligand binding, with agonists acting as allosteric potentiators of their GEF activity; promoting conformational rearrangements leading to recruitment of heterotrimeric $G$ proteins. Binding of $\mathrm{G}$ proteins to agonist-occupied GPCRs sponsors exchange of GDP for GTP. The GTPbound active form of $\mathrm{G} \alpha$ is then released and is able to interact with downstream effectors to transmit the signal. G $\alpha$ proteins contain two major structural domains, a Ras-like domain and an $\alpha$-helical domain. GPCR-G protein binding and nucleotide exchange happen via several identifiable steps. There is thought to be an initial interaction between the agonist-bound receptor and the surface of the Ras-like domain at which point the Ras-like and $\alpha$-helical domains may open. Nucleotide release then requires both an open conformation between the Ras-like and $\alpha$-helical domains of $\mathrm{G}$ protein and engagement of the $\alpha 5$ helix by the GPCR. $\alpha 5$ helix engagement disrupts interactions between $G$ protein and GDP, allowing its release (e.g., Dror et al. [2]). It is not clear how GTP binding occurs to stimulate $G$ protein release, but it is assumed that the high cellular concentration of GTP and the additional interactions of $\gamma$-phosphate allow this to occur. The molecular details of this activation mechanism have been demonstrated for Gas at the $\beta 2$-adrenergic receptor and it is assumed that the same mechanisms translate to all $\mathrm{G} \alpha$ isoforms, across all GPCRs. Not only does the GPCR- $\alpha 5$ helix interaction appear to be required for $G$ protein activation but it also has an undisputed role in GPCR-G protein specificity, as the exchange of this C-terminal fragment of one $\mathrm{G}$ protein with another confers a selectivity swap amongst GPCRs (e.g., Conklin et al. [3]). In contrast, it is relatively difficult to identify motifs in GPCRs that provide specificity for activating particular $G$ proteins and even more difficult to identify motifs that enable GPCRs to efficiently couple to more than one $\mathrm{G} \alpha$ isoform.

To address this knowledge gap, Flock et al. [1] compare conserved residues among $\mathrm{G} \alpha$ protein orthologs of 66 organisms across the fungi/metazoan group (Opisthokonta) then analyze these conserved residues against known structural data of nucleotide-bound $\mathrm{G} \alpha$ structures to filter for residues that are candidates for GPCR binding. They then filter against $G \alpha$ paralogs to identify residues that may provide specificity for GPCR binding. Lastly, the selectivity residues of Gas are mapped onto the 3 GPCR active structures that were available at the time when this manuscript was prepared (Gt peptide-bound Rhodopsin [4], mini Gas A2A [5] and Gas $\beta \gamma: \beta 2 A R$ [6]), with particular emphasis on the Gas $\beta \gamma: \beta 2 A R$ structure. They use this interaction data to develop a footprint for GPCR residues that they propose to be involved in $\mathrm{G}$ protein binding selectivity, and this selectivity is informed by sequence comparison across GPCRs using the known coupling preference (IUPHAR) and the evolutionary history (e.g., comparing closely related GPCRs that have changed $G \alpha$ coupling specificity). This is then integrated into a publicly available database.

As mentioned, at the time of preparation only one active structure with full-length Gas $\beta \gamma$ was available, 
recently, two additional active structures with full-length Gas $\beta \gamma$ have become available, that with human calcitonin receptor (CTR) [7] and that with rabbit glucagon-like peptide 1 receptor (GLP-1R) [8]. Using their database it can be seen that the $\mathrm{G} \alpha$ subunit selectivity residues indeed provide unique interfaces with these 3 receptors (Figure 1A), such that key $\mathrm{G} \alpha$ residues make some common but a number of different receptor contacts. It is unclear how these contact differences enable the differential pleiotropy exhibited by $\beta 2 A R$, which can couple to Gai when compared to CTR, and GLP-1R,

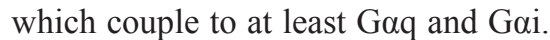
In addition, there are subtle differences in $\mathrm{G} \alpha$ conformation among these receptors, which arise from the very complex interaction that occurs across a large buried surface for both proteins.

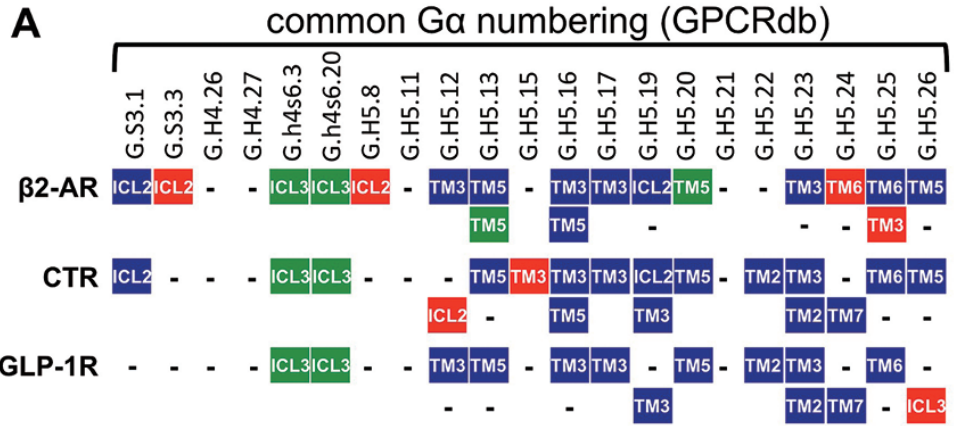

B
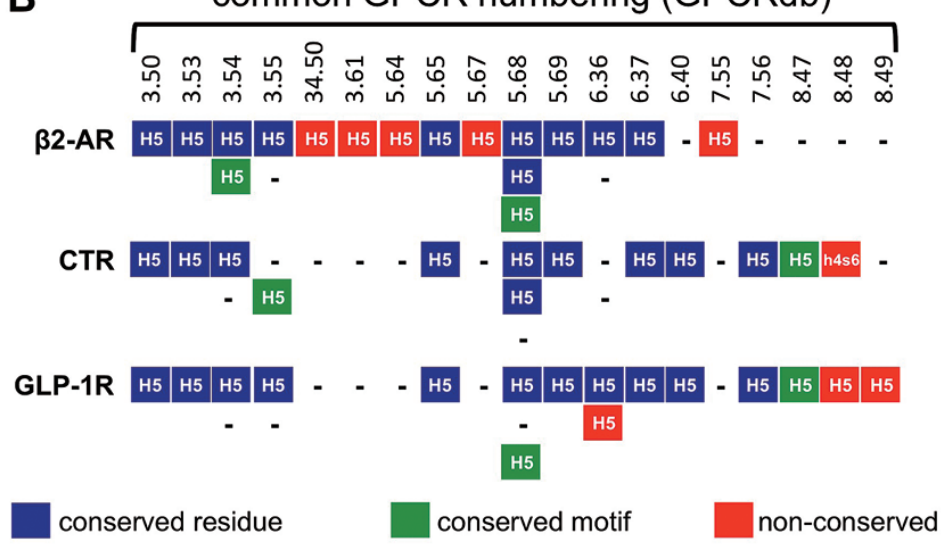

Figure 1 Selectivity determinants from GPCRdb were mapped onto Gas $\beta \gamma: \beta 2 A R$ (3sn6), Gas $\beta \gamma$ :CTR (5uz7) and G $\alpha s \beta$ :GLP-1R (5vai), and residues within $\sim 4 \AA$ and showing an appropriate interaction were manually annotated (other residues are not shown). In A, residues from the $\mathrm{G} \alpha$ interface are mapped against the 3 receptors. $\mathrm{G} \alpha$ positions are labeled using the common $\mathrm{G} \alpha$ numbering system (GPCRdb) where $\mathrm{S} 3$ is $\beta$-strand $3, \mathrm{~h} 4 \mathrm{~s} 6$ is the loop between helix 4 and $\beta$-strand 6 and $\mathrm{H} 5$ is helix 5 (all in the Ras-like domain). These are shown against interacting residues in the 3 GPCRs; in blue are GPCR residues that have corresponding positions (according to GPCRdb), green are GPCR residues in corresponding secondary structures but at alternative positions and red illustrates unique GPCR residues. In $B$, residues from the $G$ protein-coupling pocket are mapped against GPCRs (only interacting residues are shown); the color scheme is the same as $\mathbf{A}$ but applies to Gas. Pocket residues are numbered according to GPCRdb in which the first (single) number indicates the transmembrane helix or, in the case of 2 numbers, the loop connecting 2 helices. The number to the right of the decimal indicates the position of this residue relative to the most conserved helix residue, which is given the value of 50 .
These conformational differences may be important for differential $G$ protein coupling and are likely to alter nucleotide exchange $[9,10]$. The GPCR selectivity determinants identified in the paper largely identify common residues that are present in the $\alpha 5$ helix of Gas (Figure 1B). This is due to the fact that other residues at the GPCR-Ga interface do not appear to be shared among these 3 receptors. Integration of this new structural data into the database should provide even better texture to the information that it provides.

Collectively, the analysis performed and the database that evolves out of the paper has high utility; these provide a springboard for analysis of coupling data and provide direction for design of experiments to understand how coupling specificity and pleiotropy may have arisen and may operate.

\section{Sebastian GB Furness ${ }^{1}$,} Patrick M Sexton ${ }^{1}$

${ }^{1}$ Drug Discovery Biology and Department of Pharmacology, Monash Institute of Pharmaceutical Sciences, Monash University, Parkville, 3052 Victoria, Australia

Correspondence: Sebastian GB Furness ${ }^{\mathrm{a}}$, Patrick M Sexton ${ }^{\mathrm{b}}$

${ }^{a}$ E-mail: sebastian.furness@monash.edu

bE-mail: patrick.sexton@monash.edu

\section{References}

1 Flock T, Hauser AS, Lund N, et al. Nature 2017; 545:317-322.

2 Dror RO, Mildorf TJ, Hilger D, et al. Science 2015; 348:1361-1365.

3 Conklin BR, Farfel Z, Lustig KD, et al. Nature 1993; 363:274-276.

4 Scheerer P, Park JH, Hildebrand PW, et al. Nature 2008; 455:497-502.

5 Carpenter B, Nehmé R, Warne T, et al. Nature 2016; 536:104-107.

6 Rasmussen SGF, DeVree BT, Zou Y, et al. Nature 2011; 477:549-555.

7 Liang YL, Khoshouei M, Radjainia M, et al. Nature 2017; 546:118-123.

8 Zhang Y, Sun B, Feng D, et al. Nature 2017; 546:248-253.

9 Furness SGB, Liang Y-L, Nowell CJ, et al. Cell 2016; 167:739-749.

10 Gregorio GG, Masureel M, Hilger D, et al. Nature 2017; 547:68-73. 